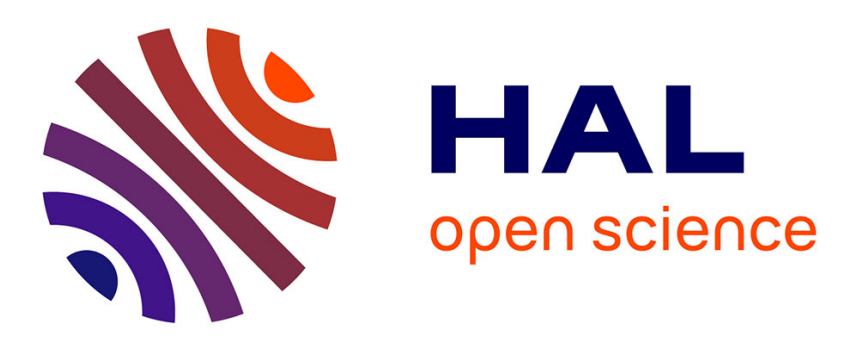

\title{
On the Use of the Multiple Scale Method in Solving 'Difficult' Bifurcation Problems
}

Angelo Luongo

\section{To cite this version:}

Angelo Luongo. On the Use of the Multiple Scale Method in Solving 'Difficult' Bifurcation Problems. 2013. hal-00904595

\section{HAL Id: hal-00904595 \\ https://hal.science/hal-00904595}

Preprint submitted on 14 Nov 2013

HAL is a multi-disciplinary open access archive for the deposit and dissemination of scientific research documents, whether they are published or not. The documents may come from teaching and research institutions in France or abroad, or from public or private research centers.
L'archive ouverte pluridisciplinaire HAL, est destinée au dépôt et à la diffusion de documents scientifiques de niveau recherche, publiés ou non, émanant des établissements d'enseignement et de recherche français ou étrangers, des laboratoires publics ou privés. 


\title{
On the Use of the Multiple Scale Method in Solving 'Difficult' Bifurcation Problems
}

\begin{abstract}
Several algorithms consisting in 'non-standard' versions of the Multiple Scale Method are illustrated for 'difficult' bifurcation problems. Preliminary, the 'easy' case of bifurcation from a cluster of distinct eigenvalues is addressed, which requires using integer power expansions, and it leads to bifurcation equations all of the same order. Then, more complex problems are studied. The first class concerns bifurcation from a defective eigenvalue, which calls for using fractional power expansions and fractional time-scales, as well Jordan or Keldysh chains. The second class regards the interaction between defective and non-defective eigenvalues. This problem also requires fractional powers, but it leads to differential equations which are of different order for the involved amplitudes. Both autonomous and parametrically excited non-autonomous systems are studied. Moreover, the transition from a codimension-3 to a codimension-2 bifurcation is explained. As a third class of problems, singular systems possessing an evanescent mass, as Nonlinear Energy Sinks, are considered, and both autonomous systems undergoing Hopf bifurcation and non-autonomous systems under external resonant excitation, are studied. The algorithm calls for a suitable combination of the Multiple Scale Method and the Harmonic Balance Method, this latter to be applied exclusively to the singular equations. Several applications are shown, to test the effectiveness of the proposed methods. They include discrete and continuous systems, autonomous, parametrically and externally excited systems.

\section{Keywords}

Bifurcation Analysis - Perturbation Methods - Defective Eigenvalues - Parametric Stabilization - Relaxation Oscillations — Nonlinear Energy Sinks
\end{abstract}

Angelo LUONGO, International Research Center M\&MoCS, University of L'Aquila - Italy, angelo.luongo@univaq.it

\section{Introduction}

Dynamical systems depend on parameters. When these are varied quasi-statically, the dynamics can, either, remain unchanged, or strongly modify. A bifurcation is said to occur at a point of the parameter space at which the dynamics abruptly changes. Here, new equilibria or cycles arise or disappear, and/or their stability changes. The bifurcation points occur on manifolds which separate the parameter space in regions in which the system possesses equivalent dynamics. Therefore bifurcations organize the scenario. The codimension of the manifold (equal to the number of independent algebraic conditions satisfied by parameters at bifurcation) is said the codimension of the bifurcation. In this paper, only bifurcations from equilibria are considered, and, although not strictly necessary, mechanical systems are addressed.

Bifurcation problems are almost universally tackled via the Center Manifold Method (CMM) and the Normal Form Theory (NFT) [1]. The CMM reduces the dimension of the dynamical system to that of the center subspace of the Jacobian (i.e. to the number of its eigenvalues having zero real part); the NFT reduces the complexity of the equations, via a quasi-identical change of coordinates, aimed to eliminate the possible largest number of nonlinear terms. The CMM is not only an algorithm, but reveals the true essence of the dynamics of a system close to bifurcation. From a geometrical point of view, it states that an attractive surface (Center Manifold, CM) exists in the (finiteor infinite-dimensional) state-space. The CM is found to be tangent at the equilibrium point to the center subspace, so that it can be tough as originated by a deformation of this subspace, caused by nonlinearities. From a mechanical point of view, the $\mathrm{CM}$ establishes a constraint among the state variables. Few of them, describing the motion in the tangent plane, participate with their own natural frequency, and therefore are said active coordinates; the remaining ones, describing motions orthogonal to the plane, do not contribute with their own frequencies, but are driven from the active coordinates, and therefore are said passive coordinates. The active coordinates are first-order quantities, while the passive coordinates are second- or higherorder quantities. However, they cannot be neglected, since they contribute to determine stability and to organize the dynamics.

A completely different approach to derive the normal form of the bifurcation equations, is offered by the Multiple Scale Method (MSM). Although this algorithm is very popular in nonlinear dynamics [2], it is less known, or even looked at with suspicion, in the bifurcation community. This standpoint is probably due to the fact the, while the CMM is based on a theorem, the MSM is a heuristic approach, based on formal series expansions. On the other hand, it has been shown in liter- 
ature, that the MSM is computationally more convenient, and furnishes the same results of the classical approach $[3,4,5,6]$. Namely, it supplies reduced equations of motion without describing the center manifold in advance; moreover, it furnishes bifurcation equations directly in normal form. Thus, it performs simultaneously the two operations of the classical approach.

However, there exist 'difficult bifurcations' for which it has been erroneously believed in literature, that the MSM (or, more in general, any perturbation method) fails in determining the bifurcation equations. In spite of this opinion, the author and his co-workers showed that, to be successful, the MSM must only be adapted to the problem; once again the method turned out to be computationally competitive with other methods.

Here, we will discuss three classes of 'difficult' problems:

1. Defective autonomous systems, in which the Jacobian operator at bifurcation does not posses a complete system of eigenvectors [7, 8];

2. Partially defective autonomous systems, in which only some of the center eigenvalues are defective [9]; or, Hamiltonian systems close to divergence, stabilized by resonant parametric excitation [10];

3. Singular, partially defective systems, in which an evanescent mass coefficient exists, either autonomous [11] or externally excited [12].

All these problems require special treatments that will be sketched ahead; additional details can be found in the cited papers.

\section{Bifurcation analysis from a cluster of dis- tinct eigenvalues: the 'easy' case}

We introduce the MSM referring to the simplest case in which the center eigenvalues are distinct, so that their associated eigenvectors form a complete basis for the center subspace (nondefective cases, see also [13, 14, 15]).

\subsection{Finite-dimensional systems}

A finite-dimensional, autonomous, dynamical system is considered, whose nondimensional equations of motion, in state-form and expanded around the trivial equilibrium point $\mathbf{x}=\mathbf{0}$, read:

$$
\dot{\mathbf{x}}=\mathbf{J}(\mu) \mathbf{x}+\mathbf{n}_{2}(\mathbf{x}, \mathbf{x})+\mathbf{n}_{3}(\mathbf{x}, \mathbf{x}, \mathbf{x})+\ldots
$$

Here $\mathbf{x}$ is the $n$-dimensional state-vector, $\mathbf{J}(\mu):=\mathbf{J}_{0}+\mu \mathbf{J}_{1}$ is the Jacobian matrix at the origin, depending on a vector of bifurcation parameters $\mu$, and $\mathbf{n}_{i}(\cdot)$ are vector-valued homogeneous polynomials of degree $i=2,3, \ldots$; moreover, the dot denotes differentiation with respect the time $t$. It is assumed that $\mu=\mathbf{0}$ is a bifurcation point, at which the Jacobian $\mathbf{J}(\mathbf{0})=\mathbf{J}_{0}$ admits one or more distinct center eigenvalues $\lambda_{c}:=\left(\lambda_{c j}\right)$. To exemplify, in a codimension-3 zero-Hopf-Hopf bifurcation (ZHH, see [6]) there are five eigenvalues in the center spectrum, namely $\lambda_{c}=\left(0, \pm i \omega_{1}, \pm i \omega_{2}\right)$, while the remaining stable eigenvalues have negative real part. We want to analyze the dynamics of the system around the equilibrium point $\mathbf{x}=\mathbf{0}$, when $\mu$ is varied in a ball of radius $0<\varepsilon \ll 1$, centered at $\mu=\mathbf{0}$.

First, in order a perturbation parameter appear in the equations, a rescaling is performed, namely $\mathbf{x} \rightarrow \varepsilon \mathbf{x}, \mu \rightarrow \varepsilon \mu$. Then, according to the MSM, $\mathbf{x}(t)=\mathbf{x}\left(t_{0}(t), t_{1}(t), t_{2}(t), \ldots\right)$ is taken, where $t_{k}:=\varepsilon^{k} t$ are independent time-scales. Therefore, by the chain rule, $d / d t=\sum_{k=0}^{\infty} \varepsilon^{k} d_{k}$, where $d_{k}:=\partial / \partial t_{k}$. Finally, the state variables are expanded as $\mathbf{x}=\sum_{k=0}^{\infty} \varepsilon^{k} \mathbf{x}_{k}$, with $\mathbf{x}_{k}$ unknowns. When all these rescaling/expansions are substituted into the $\mathrm{Eq}(1)$ and terms with the same power of $\varepsilon$ separately equated to zero, a set of linear perturbation equations is drawn, namely:

$$
\begin{aligned}
\left(d_{0}-\mathbf{J}_{0}\right) \mathbf{x}_{0}= & \mathbf{0} \\
\left(d_{0}-\mathbf{J}_{0}\right) \mathbf{x}_{1}= & -d_{1} \mathbf{x}_{0}+\mu \mathbf{J}_{1} \mathbf{x}_{0}+\mathbf{n}_{2}\left(\mathbf{x}_{0}, \mathbf{x}_{0}\right) \\
\left(d_{0}-\mathbf{J}_{0}\right) \mathbf{x}_{2}= & -\left(d_{2} \mathbf{x}_{0}+d_{1} \mathbf{x}_{1}\right)+\mu \mathbf{J}_{1} \mathbf{x}_{1}+\mathbf{n}_{3}\left(\mathbf{x}_{0}, \mathbf{x}_{0}, \mathbf{x}_{0}\right) \\
& +2 \mathbf{n}_{2}\left(\mathbf{x}_{0}, \mathbf{x}_{1}\right)
\end{aligned}
$$

The solution to Eq (2-a), when $t \rightarrow \infty$, reads (generating solution):

$$
\begin{aligned}
\mathbf{x}_{0}= & A_{0}\left(t_{1}, t_{2}, \ldots\right) \mathbf{u}_{0}+A_{1}\left(t_{1}, t_{2}, \ldots\right) \mathbf{u}_{1} \mathrm{e}^{i \omega_{1} t_{0}} \\
& +A_{2}\left(t_{1}, t_{2}, \ldots\right) \mathbf{u}_{2} \mathrm{e}^{i \omega_{2} t_{0}}+\text { c.c. }
\end{aligned}
$$

where $\mathbf{u}_{j}$ are the (right) eigenvectors of $\mathbf{J}_{0}$ which span its center subspace, i.e. $\left(\mathbf{J}_{0}-\lambda_{c j} \mathbf{I}\right) \mathbf{u}_{j}=\mathbf{0}$; the real $A_{0}$ and the complex $A_{1}, A_{2}$ are amplitudes depending on the slower time-scales; finally c.c. means complex conjugates. Note that $\mathrm{Eq}(3)$ states that the leading part of the motion belongs to the center subspace, according to the CM theory.

When the generating solution is substituted in Eq (2-b), several combinations among the eigenvalues appear in the 'forcing' terms, namely $\left(0, \omega_{1}, \omega_{2} ; 2 \omega_{1}, 2 \omega_{2}, \omega_{1} \pm \omega_{2}\right)$. To avoid that $\mathbf{x}_{1}$ diverges on the $t_{0}$-scale, thus making the series not uniformly valid, resonant terms must be removed on the right hand member, by solvability conditions requiring the known term is orthogonal to the left eigenvectors $\mathbf{v}_{j}$ of $\mathbf{J}_{0}$, having equal eigenvalue. In selecting the resonant terms, possible internal resonance must be accounted for; for example the 'forcing' frequency $\omega_{1}-\omega_{2}$ is resonant with the natural frequency $\omega_{2}$ if $\omega_{1}=2 \omega_{2}$. The solvability conditions, since they always involve $d_{1} \mathbf{x}_{0}$, are of the type:

$$
d_{1} A_{j}=f_{1 j}\left(A_{0}, A_{1}, A_{2} ; \mu\right), \quad j=0,1,2
$$

i.e., they are first-order differential equations on the $t_{1}$-scale. By solving Eq (2-b) $\mathbf{x}_{1}$ is determined, to within some arbitrary quantity, that can be chosen by requiring $\mathbf{x}_{1}$ is orthogonal to the center subspace. Thus, $\mathbf{x}_{1}$ describes the main contribution of the passive coordinates, in the $\mathrm{CM}$ view.

The procedure can be carried out to higher-orders, to evaluate $d_{2} A_{k}, d_{3} A_{k}, \ldots$. At the end, all these partial differential equations can be recombined in first-order ordinary differential equations, according to $\dot{A}_{j}=\sum_{k=0}^{\infty} \varepsilon^{k} d_{k} A_{j}$, i.e.:

$$
\dot{A}_{j}=f_{j}\left(A_{0}, A_{1}, A_{2} ; \mu\right), \quad j=0,1,2
$$

where the perturbation parameter has been reabsorbed via a backward rescaling. These are the bifurcation equations sought for; they state that the (asymptotic) motion occurs on a (fivedimensional) manifold having the same dimension of the center 
subspace. They are found to coincide with those furnished by the CMM, and already appear in their normal form.

The lower-codimension cases of zero-Hopf (ZH) and HopfHopf (HH) bifurcations can easily be obtained from the previous analysis by zeroing the amplitude of the missing mode, i.e.:

$$
\dot{A}_{j}=\left\{\begin{array}{lll}
f_{j}\left(A_{0}, A_{1} ; \mu\right) & j=0,1 & \text { for ZH } \\
f_{j}\left(A_{1}, A_{2} ; \mu\right) & j=1,2 & \text { for } \mathrm{HH}
\end{array}\right.
$$

\subsection{Infinite-dimensional systems}

The method illustrated above can be straightforwardly extended to infinite-dimensional systems $[16,17,18]$. The relevant equations of motion read:

$$
\dot{\mathbf{w}}=\mathbf{L}(\mu) \mathbf{w}+\mathbf{n}_{2}(\mathbf{w}, \mathbf{w})+\mathbf{n}_{3}(\mathbf{w}, \mathbf{w}, \mathbf{w})+\ldots
$$

where $\mathbf{w}=\mathbf{w}(\mathbf{x}, t)$ are state variables defined in a domain $\Omega$, $\mathbf{L}:=\mathbf{L}_{0}+\mu \mathbf{L}_{1}$ is a linear differential operator, and $\mathbf{n}_{i}(\cdot)$ are vector-valued homogeneous polynomial of degree $i=2,3, \ldots$. All the operators include field equations and boundary conditions.

By proceeding as for discrete systems, the following perturbation equations are drawn:

$$
\begin{aligned}
\left(d_{0}-\mathbf{L}_{0}\right) \mathbf{w}_{0}= & \mathbf{0} \\
\left(d_{0}-\mathbf{L}_{0}\right) \mathbf{w}_{1}= & -d_{1} \mathbf{w}_{0}+\mu \mathbf{L}_{1} \mathbf{w}_{0}+\mathbf{n}_{2}\left(\mathbf{w}_{0}, \mathbf{w}_{0}\right) \\
\left(d_{0}-\mathbf{L}_{0}\right) \mathbf{w}_{2}= & -\left(d_{2} \mathbf{w}_{0}+d_{1} \mathbf{w}_{1}\right)+\mu \mathbf{L}_{1} \mathbf{w}_{1} \\
& +\mathbf{n}_{3}\left(\mathbf{w}_{0}, \mathbf{w}_{0}, \mathbf{w}_{0}\right)+2 \mathbf{n}_{2}\left(\mathbf{w}_{0}, \mathbf{w}_{1}\right)
\end{aligned}
$$

The steady generating solution, in the Hopf-Hopf-divergence case, is:

$$
\begin{aligned}
\mathbf{x}_{0}= & A_{0}\left(t_{1}, t_{2}, \ldots\right) \phi_{0}(\mathbf{x})+A_{1}\left(t_{1}, t_{2}, \ldots\right) \phi_{1}(\mathbf{x}) \mathrm{e}^{i \omega_{1} t_{0}} \\
& +A_{2}\left(t_{1}, t_{2}, \ldots\right) \phi_{2}(\mathbf{x}) \mathrm{e}^{i \omega_{2} t_{0}}+\text { c.c. }
\end{aligned}
$$

where $\phi_{j}(\mathbf{x})$ are the (right) eigenvectors of $\mathbf{L}_{0}$ which span its center subspace, i.e. $\left(\mathbf{L}_{0}-\lambda_{c j} \mathbf{I}\right) \phi_{j}=\mathbf{0}$.

When this solution is substituted into the next perturbation equation, resonant terms appear. To avoid that $\mathbf{w}_{1}$ diverges on the $t_{0}$-scale, each resonant terms must be made orthogonal to the left eigenvector $\psi_{j}(\mathbf{x})$ of equal eigenvalue. These latter are solutions of the adjoint homogeneous problem $\left(\mathbf{L}_{0}^{\star}-\bar{\lambda}_{c j} \mathbf{I}\right) \psi_{j}=$ $\mathbf{0}$, where $\mathbf{L}_{0}^{\star}$ is the adjoint operator (including adjoint boundary conditions), supplied by the Green (or bilinear) Identity:

$$
\int_{\Omega} \bar{\psi}^{T} \mathbf{L}_{0} \phi d \Omega=\int_{\Omega} \bar{\phi}^{T} \mathbf{L}_{0}^{\star} \psi d \Omega
$$

Once the solvability condition is systematically enforced at any steps, partial time-differential equations for the amplitudes, $d_{k} A_{j}=f_{k j}\left(A_{0}, A_{1}, A_{2} ; \mu\right), k=1,2, \ldots$, are drawn. These equations, when are recombined to come back to the true time, turn out to be formally equal to Eqs (5). Thus, an infinitedimensional system is reduced to a 5-dimensional system.

We conclude, by the MSM, that the dimensions of the bifurcation equations do not depend on the dimensions of the original system, but rather of those of its center sub-space, as stated by the CM theory.

As an example, an elastic cantilevered beam, grounded with a visco-elastic device applied at the free end, and there loaded by a follower force, was studied in [18]. The scenarios relevant to $\mathrm{HH}$ and ZH bifurcations are shown in Fig 1, in the plane of the control parameters $\mu=(\beta, \gamma)$, where $\beta$ is a stiffness coefficient and $\gamma$ a load parameter. The bifurcation charts display the qualitative dynamics occurring in the two-dimensional phasespace $\left\{a_{j}\right\}:=\left\{\left|A_{j}\right|\right\}$, in each region of the parameter space bounded by bifurcation loci.

\section{Defective bifurcations}

\subsection{Finite-dimensional systems}

Let us assume that the Jacobian matrix is defective at the bifurcation, i.e. the eigenvectors associated with the center eigenvalues are less than their algebraic multiplicity, so that the basis is incomplete. For example, let $\lambda_{c}=(0,0)$ be a doublezero eigenvalue, with just a (proper) eigenvector $\mathbf{u}_{0}$ associated. Then a Takens-Bogdanov (or double-zero, DZ) bifurcation, of codimension-2, is said to occur.

To complete the basis, a (index-2) generalized eigenvector must be associated, so that a Jordan chain $\left(\mathbf{u}_{01}, \mathbf{u}_{02}\right)$, with $\mathbf{u}_{01}:=\mathbf{u}_{0}$ must be built-up by solving the sequence of algebraic problems:

$$
\begin{aligned}
\mathbf{J}_{0} \mathbf{u}_{01} & =\mathbf{0} \\
\mathbf{J}_{0} \mathbf{u}_{02} & =\mathbf{u}_{01}
\end{aligned}
$$

under arbitrarily chosen normalization conditions. Now, it is known from algebra, that, said $\mathbf{v}_{0}$ the unique proper left eigenvector (satisfying $\mathbf{J}_{0}^{T} \mathbf{v}_{0}=\mathbf{0}$ ), it results that $\mathbf{v}_{0}^{T} \mathbf{u}_{01}=0$. From a geometrical point of view, since $\mathbf{v}_{0}$ spans the kernel of the adjoint operator $\mathbf{J}_{0}^{T}, \mathbf{u}_{01}$ is in the range of the operator. Only the highest-index of the chain (in our case $\mathbf{u}_{02}$ ) has a component out the the range, namely $\mathbf{v}_{0}^{T} \mathbf{u}_{02} \neq 0$.

Due to these properties, the integer power expansions used in the standard approach fail, since, in removing resonant terms, $\mathbf{v}_{0}^{T} d_{1} \mathbf{x}_{0}=0$ is found, so that the left hand member of Eq (4) disappears! To overcome the drawback, fractional series expansions must be used, in order to create intermediate perturbation equations able to generate the highest vector of the chain, when the resonant terms appear first [7]. Thus, if the eigenvalue has multiplicity $m$, fractional powers $\varepsilon^{1 / m}$ must be used; here we discuss the simplest case $m=2$.

With the same rescaling as before, we introduce fractional time scales $t_{0}, t_{1 / 2}, t_{1}, \ldots$, so that $d / d t=\sum_{k=0}^{\infty} \varepsilon^{k / 2} d_{k / 2}$; moreover, we expand the state variables as $\mathbf{x}=\sum_{k=0}^{\infty} \varepsilon^{k / 2} \mathbf{x}_{k / 2}$. Thus we obtain the following perturbation equations:

$$
\begin{aligned}
& \left(d_{0}-\mathbf{J}_{0}\right) \mathbf{x}_{0}=\mathbf{0} \\
& \left(d_{0}-\mathbf{J}_{0}\right) \mathbf{x}_{1 / 2}=-d_{1 / 2} \mathbf{x}_{0} \\
& \left(d_{0}-\mathbf{J}_{0}\right) \mathbf{x}_{1}=-\left(d_{1} \mathbf{x}_{0}+d_{1 / 2} \mathbf{x}_{1 / 2}\right)+\mu \mathbf{J}_{1} \mathbf{x}_{0}+\mathbf{n}_{2}\left(\mathbf{x}_{0}, \mathbf{x}_{0}\right)
\end{aligned}
$$

and so on. 


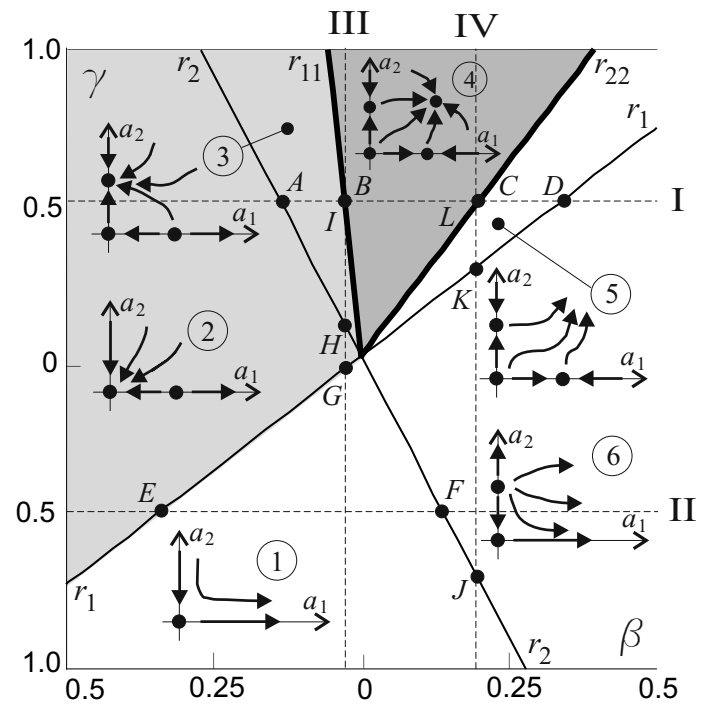

(a)

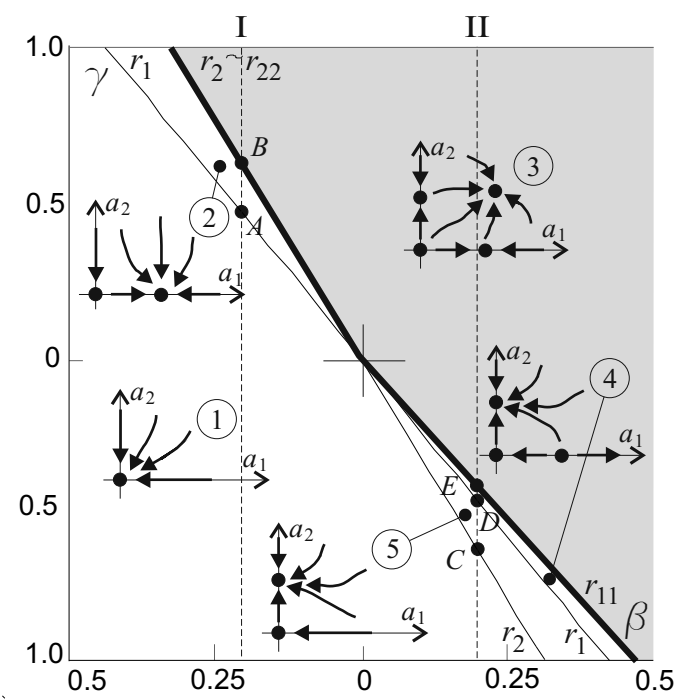

(b)

Figure 1. Bifurcation charts for an elastic beam under follower force [18]: Hopf-Hopf bifurcation, (b) zero-Hopf bifurcation.

The generating solution is:

$$
\mathbf{x}_{0}=A_{0}\left(t_{1 / 2}, t_{1}, \ldots\right) \mathbf{u}_{01}
$$

where $a$ real. It could be erroneously believed, at this level, that the leading motion takes place on a one-dimensional manifold, since the generalized eigenvector has not taken into account (as, instead, this is the case in the CMM); we will show soon, however, that this is not the case.

When the generating solution is substituted in Eq (12-b), since the 'known' term $-\left(d_{1 / 2} A_{0}\right) \mathbf{u}_{01}$ belongs to the range of the operator, no solvability is required and the solution reads $\mathbf{x}_{1 / 2}=-\left(d_{1 / 2} A_{0}\right) \mathbf{u}_{02}$. Note that the highest-index eigenvector of the chain appeared, and that, due to the lack of solvability conditions, $\left(d_{1 / 2} A_{0}\right)$ is still unknown.

With the previous results used in Eq (12-b), 'resonant' 0frequency terms appear, generated by the last two terms in the equation. However, now $d_{1 / 2} \mathbf{x}_{1 / 2}=-\left(d_{1 / 2}^{2} A_{0}\right) \mathbf{u}_{02}$ is available to remove them, since it is out of the range of the operator. This solvability condition is of the type:

$$
d_{1 / 2}^{2} A_{0}=f_{1 / 2}\left(A_{0} ; \mu\right)
$$

When further steps are performed, and the solvability conditions reconstituted in a whole, the following bifurcation equation is recovered:

$$
\ddot{A}_{0}=f\left(A_{0}, \dot{A}_{0} ; \mu\right)
$$

Note that this is a second-order ordinary differential equation in the amplitude $A_{0}$; therefore, the leading motion occurs on a two-dimensional manifold, as predicted by the CMM.

\subsection{Infinite-dimensional systems}

When the system is infinite-dimensional, the perturbation analysis closely follows that for finite-dimensional systems $[19,20$,
21, 22]. However, due to the occurrence of defective eigenvalues, we need to extend to continuous systems the concept of Jordan chain. This task has been accomplished by Keldysh [23, 24], who defined Keldysh chains for one-dimensional systems, similarly to the Jordan chains. Thus, if $\lambda_{c}=(0,0)$ is a double-zero eigenvalue, a Keldysh chain $\left(\phi_{01}, \phi_{02}\right)$ is defined, satisfying the equations:

$$
\begin{aligned}
& \mathbf{L}_{0} \phi_{01}=\mathbf{0} \\
& \mathbf{L}_{0} \phi_{02}=\phi_{01}
\end{aligned}
$$

where $\phi_{01}$ is a proper eigenvector, and $\phi_{02}$ an index-2 generalized eigenvector. The same orthogonality conditions observed for the algebraic system holds, i.e. $\int_{\Omega} \bar{\psi}^{T} \phi_{01} d \Omega=0$, $\int_{\Omega} \bar{\psi}^{T} \phi_{02} d \Omega \neq 0$, where $\psi$ is the proper eigenvector which spans the kernel of $\mathbf{L}_{0}^{\star}$.

By repeating the former analysis,we find $\mathbf{w}=\mathbf{w}_{0}+\varepsilon^{1 / 2} \mathbf{w}_{1}+$ ..., i.e., with $\varepsilon$ reabsorbed:

$$
\mathbf{w}=A_{0}\left(t_{1 / 2}, t_{1}, \ldots\right) \phi_{01}-\dot{A}_{0} \phi_{02}+\ldots
$$

where the evolution of the real amplitude is governed by the bifurcation equation (15).

As an example, when a visco-elastic externally damped cantilevered beam is loaded at the tip by a follower $\mu$ and a gravitational $v$ force, it undergoes a double-zero bifurcation for suitable combinations of the parameters [21,22]. The relevant bifurcation chart is shown in Fig 2 for two different damping combinations, which display a non-catastrophic or catastrophic behavior (i.e. with or without an attractor in each region).

\section{Interaction between defective and non- defective eigenvalues}

\subsection{Autonomous systems}

We saw that distinct eigenvalues call for integer power expansions, while defective eigenvalues require fractional expan- 


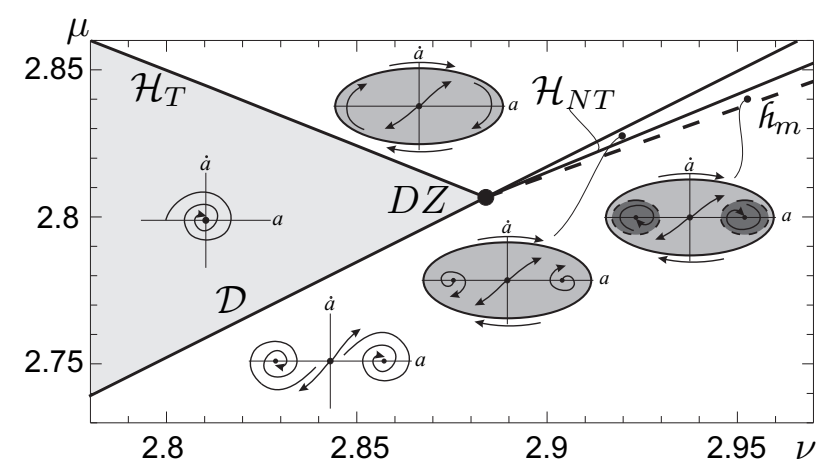

(a)

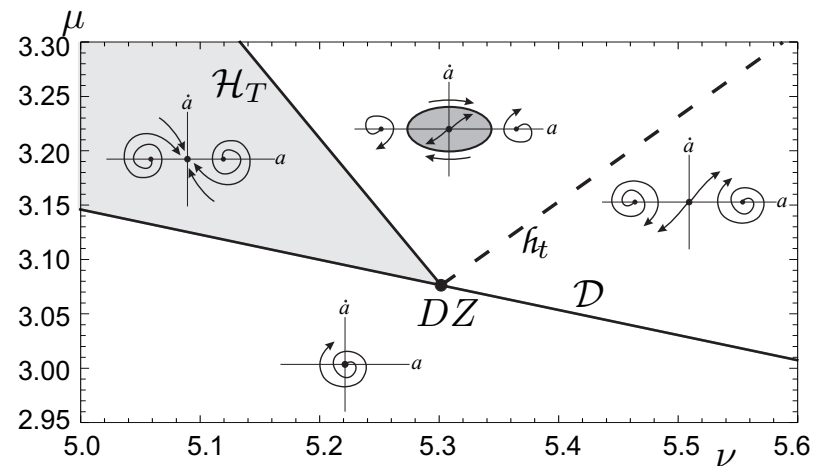

(b)

Figure 2. Bifurcation chart around a double-zero bifurcation point for a visco-elastic beam under gravitational and follower forces [21]: (a) non-catastrophic, (b) catastrophic behavior.

sions. What happens when eigenvalues of the two types interact? An example of such an occurrence is offered by the double-zero/Hopf bifurcation (DZH, see [9]). In this case, $\lambda_{c}=\left(0,0, \pm i \omega_{1}\right)$, and the associated auto-space is spanned by $\left(\mathbf{u}_{01}, \mathbf{u}_{02}, \mathbf{u}_{1}, \overline{\mathbf{u}}_{1}\right)$, where $\left(\mathbf{u}_{01}, \mathbf{u}_{02}\right)$ is a real Jordan chain, and $\left(\mathbf{u}_{1}, \overline{\mathbf{u}}_{1}\right)$ are complex conjugate complex eigenvalues. Since the defective eigenvalue $(0,0)$ calls for power expansions of $\varepsilon^{1 / 2}$ and the non-defective eigenvalues $\left(+i \omega_{1},-i \omega_{1}\right)$ for integer powers of $\varepsilon$, we chose the stronger condition, and expand in series of $\varepsilon^{1 / 2}$; consistently, we introduce time-scales multiple of the same power. As a result, we again obtain the perturbation equations (12).

The generating solution, in this case, reads:

$$
\mathbf{x}_{0}=A_{0}\left(t_{1 / 2}, t_{1}, \ldots\right) \mathbf{u}_{01}+A_{1}\left(t_{1 / 2}, t_{1}, \ldots\right) \mathbf{u}_{1} \mathrm{e}^{i \omega_{1} t_{0}}+\text { c.c. }
$$

where $A_{0}$ is real and $A_{1}$ is complex. When this is substituted in the $\mathrm{Eq}(12-\mathrm{b})$, this latter becomes:

$$
\left(d_{0}-\mathbf{J}_{0}\right) \mathbf{x}_{1 / 2}=-\left(d_{1 / 2} A_{0}\right) \mathbf{u}_{01}-\left(d_{1 / 2} A_{1}\right) \mathbf{u}_{1} \mathrm{e}^{i \omega_{1} t_{0}}+\text { c.c. }
$$

To remove secular terms, the 0 -frequency 'known term' must be rendered ortogonal to the left proper eigenvector $\mathbf{v}_{0}$, and the $\omega_{1}$-frequency term to the left proper eigenvector $\mathbf{v}_{1}$. However, due to the properties of the Jordan chains, while $\overline{\mathbf{v}}_{1}^{T} \mathbf{u}_{1} \neq 0$, it is $\mathbf{v}_{0}^{T} \mathbf{u}_{01}=0$; consequently, $d_{1 / 2} A_{1}=0$ but $d_{1 / 2} A_{0}$ is undetermined at this order. Therefore, as expected, $A_{1}$ follows the rule for non-defective amplitudes, while $A_{0}$ behaves as defective amplitudes. However, this is not a general rule, since, due to the interaction, this simple scheme does not hold at higher-orders. Moreover, it has been explained in [9] that, differently from the previous cases, in which the complementary solution of each perturbation equation can be ignored, here, in order to avoid inconsistencies, some of them must be taken into account. By skipping this discussion for the sake of brevity, we find $\mathbf{x}_{1 / 2}=-\left(d_{1 / 2} A_{0}\right) \mathbf{u}_{02}$. By going one step further, we obtain solvability conditions of the type:

$$
\begin{aligned}
& d_{1 / 2}^{2} A_{0}=f_{1 / 2,0}\left(A_{0}, A_{1} ; \mu\right) \\
& d_{2} A_{1}=f_{1 / 2,1}\left(A_{0}, A_{1} ; \mu\right)
\end{aligned}
$$

When the analysis is carried out to the higher-orders and the solvability conditions reconstituted, the following bifurcation equations are finally obtained:

$$
\begin{aligned}
& \ddot{A}_{0}=f_{0}\left(A_{0}, \dot{A}_{0}, A_{1} ; \mu\right) \\
& \dot{A}_{1}=f_{1}\left(A_{0}, \dot{A}_{0}, A_{1} ; \mu\right)
\end{aligned}
$$

They govern the dynamics of a 4-dimensional system, consistently with the dimension of the CM.

\subsection{Transition from a higher- to a lower-codimension bifurcation}

Let us consider a damped Hamiltonian systems at the divergence, under self-excitation causing Hopf bifurcation. There exist three non-hyperbolic eigenvalues $\lambda_{c}=(0, \pm i \omega)$ plus a fourth hyperbolic (stable) eigenvalue $\lambda_{s}=-\xi$ where $\xi>0$ is a damping coefficient. If the damping is large, i.e. $\xi=\mathrm{O}(1)$, we can analyze this bifurcation as a (easy) codimension- $2 \mathrm{ZH}$ bifurcation (see Fig 3-a); however, if the damping is small, i.e. $\xi=\mathrm{O}(\varepsilon)$, the system is close to a (difficult) codimension3 DZH bifurcation (see Fig 3-b). The question is: how large must be the damping in order for the fourth eigenvalue can be considered stable and not a perturbation of a zero eigenvalue? To answer the question, it needs to analyze the transition from the higher-codimension (partially-defective) to the lowercodimension (non-defective) bifurcation, by increasing $\xi$ from zero.

A paradigmatic system introduced in [9] to analyze the problem, consists of two Duffing-Van der Pol coupled oscillators, of nondimensional equations:

$$
\begin{aligned}
& \ddot{x}+x-\mu \dot{x}+g(x, y, \dot{x}, \dot{y})=0 \\
& \ddot{y}-v y-\xi \dot{y}+h(x, y, \dot{x}, \dot{y})=0
\end{aligned}
$$

where $\mu:=(\mu, v, \xi) \rightarrow \mathbf{0}$. Here, the $x$-oscillator, of finite stiffness, undergoes a Hopf bifurcation at $\mu=0$; the $y$-oscillator, of evanescent stiffness $v$ and evanescent damping $\xi$, undergoes a DZ bifurcation at $(v, \xi)=(0,0)$. The nonlinear terms $g(\cdot), h(\cdot)$ couple the two oscillators. Therefore, a DZH occurs at $\mu=\mathbf{0}$; however, if $\xi$ is allowed to become large of order 1 , a $\mathrm{ZH}$ bifurcation occurs. 


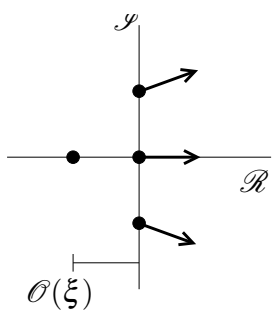

$\operatorname{Cod} 2$

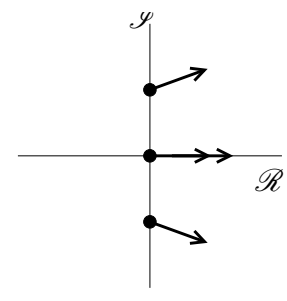

Cod 3 (a)

(b)

Figure 3. Center eigenvalues at (a) zero-Hopf bifurcations, (b) Double-zero/Hopf

The following bifurcation equations, of the type (21), were found by the MSM [9]:

$$
\begin{aligned}
& \underbrace{\ddot{A}_{0}}_{\varepsilon^{3 / 2}}=\mathscr{L}_{0}(\underbrace{v A_{0}, A_{0}^{3}, A_{1} \bar{A}_{1} A_{0}}_{\varepsilon^{3 / 2}} ; \underbrace{\xi \dot{A}_{0}}_{\varepsilon^{2}} ; \ldots) \\
& \underbrace{\dot{A}_{1}}_{\varepsilon^{3 / 2}}=\mathscr{L}_{1}(\underbrace{\mu A_{1}, A_{1}^{2} \bar{A}_{1}, A_{1} A_{0} \dot{A}_{0}}_{\varepsilon^{3 / 2}} ; \ldots)
\end{aligned}
$$

where $\mathscr{L}_{j}$ are linear operators. Here the order of the different terms is determined by the perturbation analysis, which states that $A_{0}=O\left(\varepsilon^{1 / 2}\right)$ and $A_{1}=O\left(\varepsilon^{1 / 2}\right)$; however, due to the fact that $d_{1 / 2} A_{0} \neq 0, d_{1 / 2} A_{1}=0$, it happens that $(d / d t) A_{0}=$ $\mathrm{O}\left(\varepsilon^{1 / 2} \times \varepsilon^{1 / 2}\right)$, while $(d / d t) A_{1}=\mathrm{O}\left(\varepsilon \times \varepsilon^{1 / 2}\right)$. We can conclude that the dynamic of $A_{0}$ is faster than the dynamics of $A_{1}$ (and therefore $\ddot{A}_{0}$ and $\dot{A}_{1}$ are of the same order).

When, however, $\xi$ is increased to the order- 1 , since a simple zero-eigenvalue is included in the center spectrum, the bifurcation becomes non-defective, so that no fractional powers can be involved in the perturbation analysis; therefore $(d / d t) A_{j}=$ $\mathrm{O}\left(\varepsilon \times \varepsilon^{1 / 2}\right)$ for both amplitudes. As a consequence, $\ddot{A}_{0}$ becomes of an higher-order with respect to $\dot{A}_{1}$, i.e. the dynamics of $A_{0}$ becomes slower and comparable with those of $A_{1}$. Thus, while $\ddot{A}_{0}$ jumps to the higher-order terms of Eqs (23-a), $\xi \dot{A}_{0}$ enter among the leading terms, and Eqs (23) become:

$$
\begin{aligned}
& \xi \dot{A}_{0}=-\mathscr{L}_{0}\left(v A_{0}, A_{0}^{3}, A_{1} \bar{A}_{1} A_{0} ; \ldots\right) \\
& \dot{A}_{1}=\mathscr{L}_{1}\left(\mu A_{1}, A_{1}^{2} \bar{A}_{1}, A_{1} A_{0} \dot{A}_{0} ; \ldots\right)
\end{aligned}
$$

Note that the order of the differential equations is lowered, so that the dynamics occurs on a three-dimensional manifold. These are just the bifurcation equations for the zero-Hopf bifurcation.

Numerical solutions of Eqs (23) highlighted the existence of quasi-periodic solutions which strongly affect the dynamics close to the double-zero/Hopf bifurcation. However, when the damping is increased, they move away, thus explaining the transition phenomenon. This is illustrated in Fig 4, where two bifurcation charts, for small and large damping are represented. Lines I to III denotes bifurcations from which steady solution arise (I periodic, II buckled, III periodic around a buckled equilibrium), while QP denotes quasi-periodic solutions (in which $A_{0}, A_{1}$ are periodic).

\subsection{Statically unstable systems under stabilizing para- metric excitation}

Let us consider a class of linear Hamiltonian systems, close to divergence, at which a double-zero eigenvalue occurs. It is known that, if a linear parametric excitation of frequency $\Omega$ is applied, rendering the stiffness (or the mass) time-dependent, the statically unstable system can be re-stabilized [25, 26]. The phenomenon can clearly be explained by a perturbation analysis [10], which shows how the parametric frequency combines itself with the natural frequencies of the Hamiltonian system, to produce restabilizing time-independent terms (i.e. apparent stiffnesses), which are able (if of suitable sign) to compensate the lack of stiffness of the unexcited system.

To explain the algorithm, let us consider a Hamiltonian finite-dimensional linear system, parametrically excited by a harmonic law of small amplitude $\delta$ :

$$
\dot{\mathbf{x}}=(\mathbf{J}(\mu)+\delta \mathbf{B} \cos \Omega t) \mathbf{x}
$$

and assume that $\mathbf{J}_{0}$ admits a double-zero eigenvalue, at which the unique eigenvector $\mathbf{u}_{0} \equiv \mathbf{u}_{01}$ is associated, and complex conjugate purely imaginary eigenvalues $\pm i \omega_{j}$. An exhaustive analysis of the system would require considering all the resonance conditions possibly occurring between the excitation frequency $\Omega$ and the natural frequencies $\omega_{j}$ (see [10], where a specific system was analyzed); here, we limit ourselves to the 1:1 resonant case $\Omega \simeq \omega_{1}$, by excluding any other form of resonance. In this case, the algorithm described above for DZH bifurcations of nonlinear autonomous systems also applies to this class of linear non-autonomous systems. Indeed, from a computational point of view, the harmonically-dependent coefficients have the same role played by nonlinearities in autonomous systems.

By performing the rescaling $\mu \rightarrow \varepsilon^{2} \mu, \delta \rightarrow \varepsilon \delta$, and using fractional series expansions and time-scales, we obtain the following perturbation equations:

$$
\begin{aligned}
& \left(d_{0}-\mathbf{J}_{0}\right) \mathbf{x}_{0}=\mathbf{0} \\
& \left(d_{0}-\mathbf{J}_{0}\right) \mathbf{x}_{1 / 2}=-d_{1 / 2} \mathbf{x}_{0} \\
& \left(d_{0}-\mathbf{J}_{0}\right) \mathbf{x}_{1}=-\left(d_{1} \mathbf{x}_{0}+d_{1 / 2} \mathbf{x}_{1 / 2}\right)+\frac{1}{2} \delta \mathbf{B} \mathbf{x}_{0}\left(\mathrm{e}^{i \Omega t_{0}}+\mathrm{e}^{-i \Omega t_{0}}\right)
\end{aligned}
$$

We take a generating solution made of two components: the static mode $\mathbf{u}_{01}$ and the dynamic mode $\mathbf{u}_{1}$ in resonance with the excitation (indeed, a small damping would produce the decay of all the other components). Hence, the solution to Eq (26-a) reads as Eq (18). At the next-order, Eq (26-b), the same results are found, namely $d_{1 / 2} A_{1}=0$ and $d_{1 / 2} A_{0}$ undetermined; moreover $\mathbf{x}_{1 / 2}=-\left(d_{1 / 2} A_{0}\right) \mathbf{u}_{02}$. When all these results are substituted in Eq (26-c), the parametric excitation combines with the generating solution, giving rise the forcing frequencies $\left(\Omega, \Omega \pm \omega_{j}\right)$. Since $\Omega \simeq \omega_{1}$, these frequencies are, in the order, resonant with 


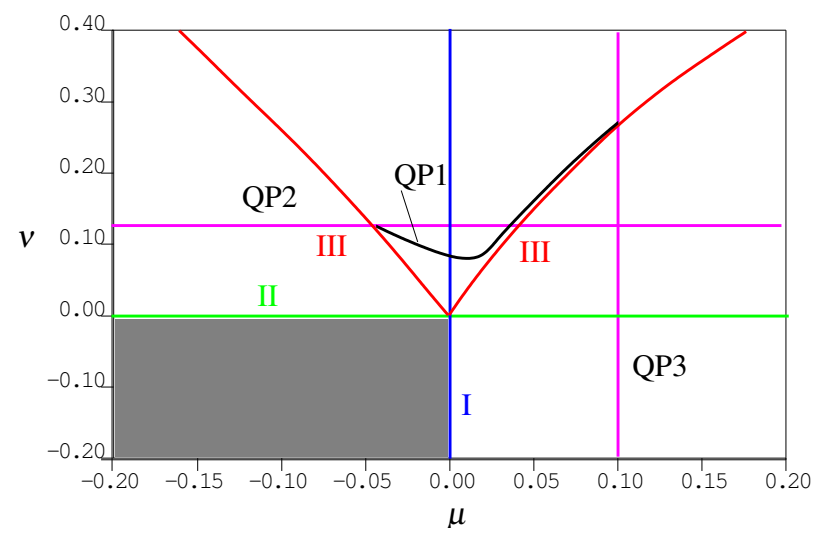

(a)

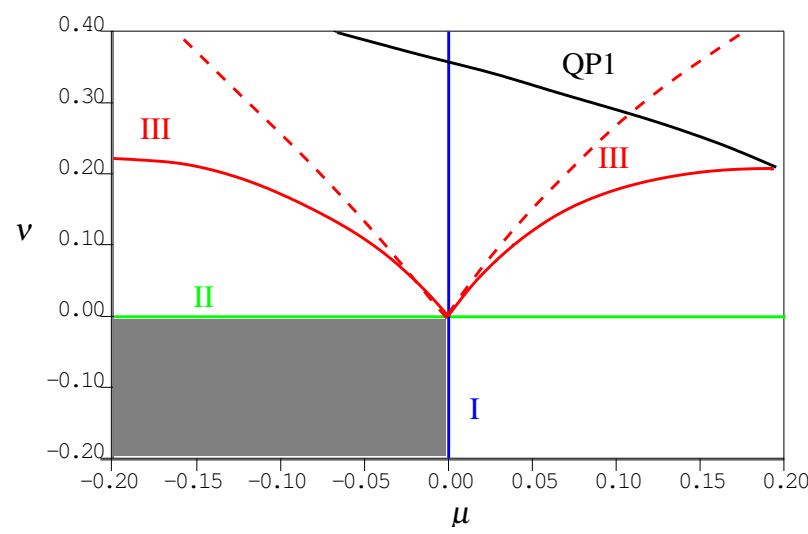

(b)

Figure 4. Bifurcation chart for a paradigmatic system, showing transition from double-zero/Hopf to zero-Hopf bifurcation [9]: (a) small damping, (b) large damping.

the dynamic mode of amplitude $A_{1}$, and the static mode of amplitude $A_{0}$, when $j=1$ is taken. Therefore, solvability, leads to the following differential equations:

$$
\begin{aligned}
& d_{1 / 2}^{2} A_{0}=\mathscr{L}_{1 / 2,0}\left(A_{1}, \bar{A}_{1} ; \delta\right) \\
& d_{2} A_{1}=\mathscr{L}_{2,1}\left(A_{0} ; \delta\right)
\end{aligned}
$$

When the procedure is carried out up to the $\varepsilon^{2}$-order, where the parameters $\mu$ have been ordered, and the solvability conditions reconstituted, the following bifurcation equations are obtained:

$$
\begin{aligned}
& \ddot{A}_{0}=\mathscr{L}_{0}\left(A_{0}, A_{1}, \bar{A}_{1} ; \delta, \mu\right) \\
& \dot{A}_{1}=\mathscr{L}_{1}\left(A_{0}, \dot{A}_{0}, A_{1}, \bar{A}_{1} ; \delta, \mu\right)
\end{aligned}
$$

which are a particular (linear) form of the more general Eqs (21).

These equations have been derived in [10] for a upright double pendulum, under gravitational forces triggering static instability, and harmonic vertical motion of amplitude $\delta$, prescribed at the support and providing the parametric excitation. The analysis led to the results displayed in Fig 5-a, where the stability region of the equilibrium position $\mathbf{x}=\mathbf{0}$ is shown in the frequency-amplitude plane. It is seen that, when the system is unexcited $(\delta=0)$, the equilibrium is unstable; however, a frequency-dependent amplitude $\delta$ exists, which stabilizes the equilibrium. The phenomenon, however, only takes place on the right of the resonance value, while no stable equilibrium exist on the left. Results have also been compared with numerical solutions based on the Floquet theory (Fig 5-b). It is seen that, while the accordance is excellent for small $\delta$, it is not good for large $\delta$. In spite of this, the MSM captures the qualitative behavior, including the existence of a narrow stability zone at higher-amplitudes.

\section{Bifurcations of singular systems: the Non- linear Energy Sinks}

\subsection{Autonomous systems undergoing Hopf bifurca- tion}

The occurrence, at the bifurcation, of the center eigenvalues $\lambda_{c}=(0,0, \pm i \omega)$ is not only an accidental (and detrimental) event, but even a desired circumstance adopted in passive control. As a matter of fact, when a mechanical system undergoes a Hopf bifurcation, e.g. triggered by wind flow forces, a control strategy recently studied in literature consists in attaching to it one (or more) essentially nonlinear oscillators (i.e. single d.o.f. devices with zero linear stiffness) having evanescent mass, said Nonlinear Energy Sinks (NES, [27, 28]). Thus, the augmented system also posseses a double zero eigenvalue. The NES works as a (well-known) Tuned Mass Damper (TMD), i.e. it is able to capture the input energy by preserving the main structure $[29,30]$; however, being essentially nonlinear, it is able to adapt its own frequency, thus working in a frequency range wider that the mono-frequent TMD. Many semi-analytical or numerical methods were proposed in literature to solve this problem, and it was common opinion that 'standard perturbation methods' do not work [31]. This is, indeed, true, but a 'non-standard' version of the MSM, combined with the Harmonic Balance Method (HBM), and therefore denoted by MS-HBM, was indeed shown to work in $[11,12]$.

To illustrate the algorithm, we consider a symmetric (odd nonlinearities) finite dimensional system of state-variables $\mathbf{x}$, with a NES attached, whose displacement is $y$ (Fig 6); the relevant equations of motion, in nondimensional form, read:

$$
\begin{aligned}
& \dot{\mathbf{x}}=\mathbf{J}(\mu) \mathbf{x}+\mathbf{n}_{3}(\mathbf{x}, \mathbf{x}, \mathbf{x})+\ldots-\left(\xi \dot{z}+\kappa z^{3}\right) \hat{\mathbf{r}} \\
& m\left(\ddot{z}-\mathbf{r}^{T} \ddot{\mathbf{x}}\right)+\xi \dot{z}+\kappa z^{3}=0
\end{aligned}
$$

where $z:=\mathbf{r}^{T} \mathbf{x}-y$ is the elongation of the NES, and $\mathbf{r}, \hat{\mathbf{r}}:=(\mathbf{0}, \mathbf{r})^{T}$ are influence coefficient vectors. Moreover, $m \ll 1$ is the NES mass, $\xi \ll 1$ the damping and $\kappa=\mathrm{O}(1)$ the nonlinear stiffness. Since $m$ is small, but different from zero, the system posses a 


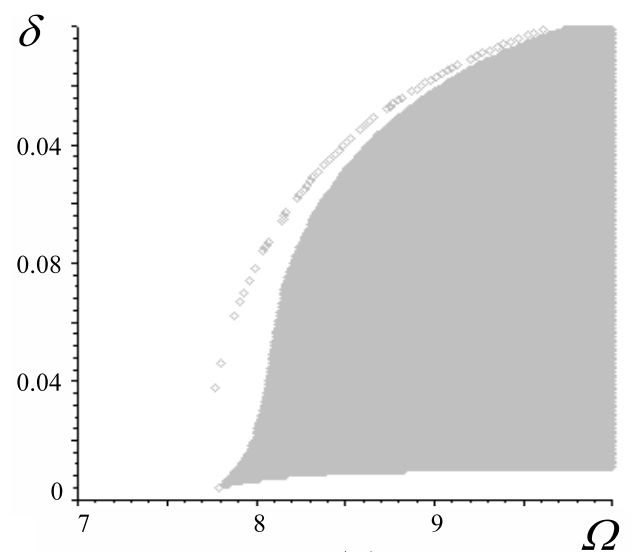

(a)

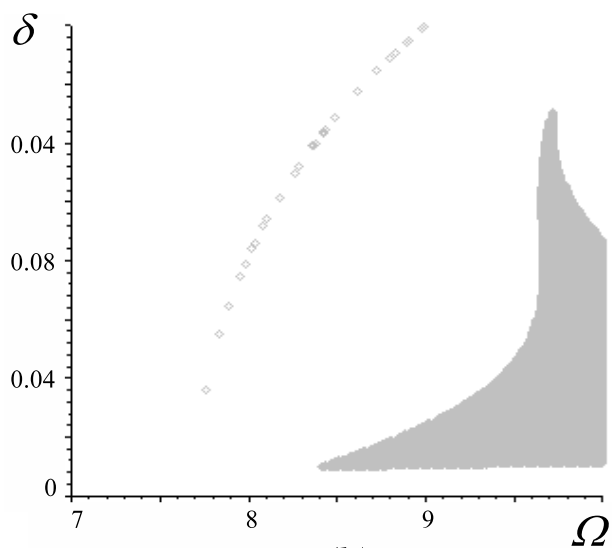

(b)

Figure 5. Stability region for the parametrically excited double pendulum close to divergence [10]: (a) MSM solution, (b) Numerical solution.

pair of (nearly) zero eigenvalues; however, since the mass is evanescent, the equations are of singular type.

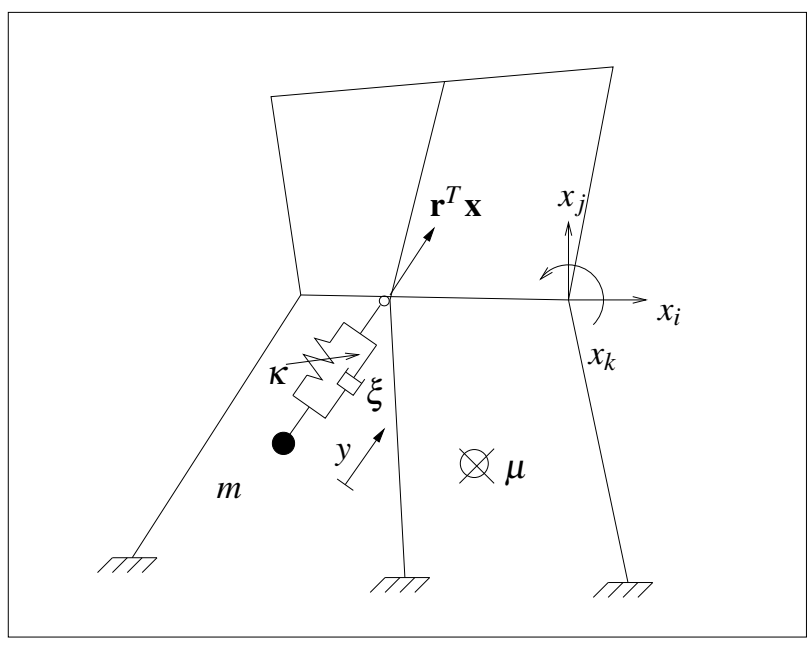

Figure 6. Sketch of a multi-d.o.f. system equipped with a NES, under normal wind flow.

To solve the problem, we first rescale the variables as $(\mathbf{x}, z) \rightarrow$ $\varepsilon^{1 / 2}(\mathbf{x}, z)$ and the parameters as $(\mu, m, \xi) \rightarrow \varepsilon(\mu, m, \xi)$; then, we expand the variables in integer series, i.e. $\mathbf{x}=\sum_{k=0}^{\infty} \varepsilon^{k} \mathbf{x}_{k}$, $z=\sum_{k=0}^{\infty} \varepsilon^{k} z_{k}$ and use integer time-scales $t_{k}:=\varepsilon^{k} t$. Thus we obtain the generating perturbation equation:

$$
\varepsilon^{0}:\left(d_{0}-\mathbf{J}_{0}\right) \mathbf{x}_{0}=\mathbf{0}
$$

the $\varepsilon^{1}$-order equations:

$$
\begin{aligned}
& \quad\left(d_{0}-\mathbf{J}_{0}\right) \mathbf{x}_{1}=-d_{1} \mathbf{x}_{0}+\mu \mathbf{J}_{1} \mathbf{x}_{0}+\mathbf{n}_{3}\left(\mathbf{x}_{0}, \mathbf{x}_{0}, \mathbf{x}_{0}\right) \\
& \quad-\left(\xi d_{0} z_{0}+\kappa z_{0}^{3}\right) \hat{\mathbf{r}} \\
& m d_{0}^{2} z_{0}+\xi d_{0} z_{0}+\kappa z_{0}^{3}=m \mathbf{r}^{T} \ddot{d}_{0}^{2} \mathbf{x}_{0}
\end{aligned}
$$

and so on. Note that, due to the fact $m$ is evanescent, the NESequation shifts at a higher order, this being the main difference with the case studied in the previous Section.
The generating solution, therefore, accounts for the uncontrolled main system. If, at $\mu=\mathbf{0}$, it undergoes a Hopf bifurcation, then:

$$
\mathbf{x}_{0}=A\left(t_{1}, t_{2}, \ldots\right) \mathbf{u}_{1} \mathrm{e}^{i \omega_{1} t_{0}}+\text { c.c. }
$$

whit $A$ a complex amplitude. When this is substituted in the NES equation (31-b), a strongly nonlinear equation in $z_{0}$, with harmonic excitation, is found. Since it cannot be solved by elementary functions, the Harmonic Balance Method (HBM) is used, by letting:

$$
z_{0}=\sum_{k=1}^{\infty} B_{0 k}\left(t_{1}, t_{2}, \ldots\right) \mathrm{e}^{i k \omega t_{0}}+c . c .
$$

where $B_{0 k}$ are complex amplitudes. The HBM furnishes a set of cubic algebraic equations of the type:

$$
\mathbf{g}_{0}\left(A, \mathbf{B}_{0}\right)=\mathbf{0}
$$

where $\mathbf{B}_{0}:=\left(B_{01}, B_{02}, \ldots\right)$. These provide an algebraic constraint between the amplitude of oscillation $A$ of the main system and the harmonic components of the NES elongation. They describe a (parameter independent) manifold in the amplitude space on which the motion occurs. The Figure 7 shows a typical manifold in the space of the moduli $\left(a, b_{01}, b_{03}\right)$ of the amplitudes $\left(A, B_{01}, B_{03}\right)$.

By substituting the $z_{0}$ solution in Eq (31-a) and removing secular terms, a differential equation governing the evolution of $A$ on the $t_{1}$-scale is found, having the form:

$$
d_{1} A=f_{1}\left(A, \mathbf{B}_{0} ; \mu\right)
$$

If the analysis is truncated at this order, the dynamics is ruled by this last equation, with $\mathbf{B}_{0}$ acting as a passive variable via the constraint (34), so that the motion is restrained to the manifold. However, if this latter exhibits turning point with respect to $A$ (as it occurs in Fig. 7), the motion cannot cross them, since $\dot{A}$ is generally different from zero there; hence, the equations break 


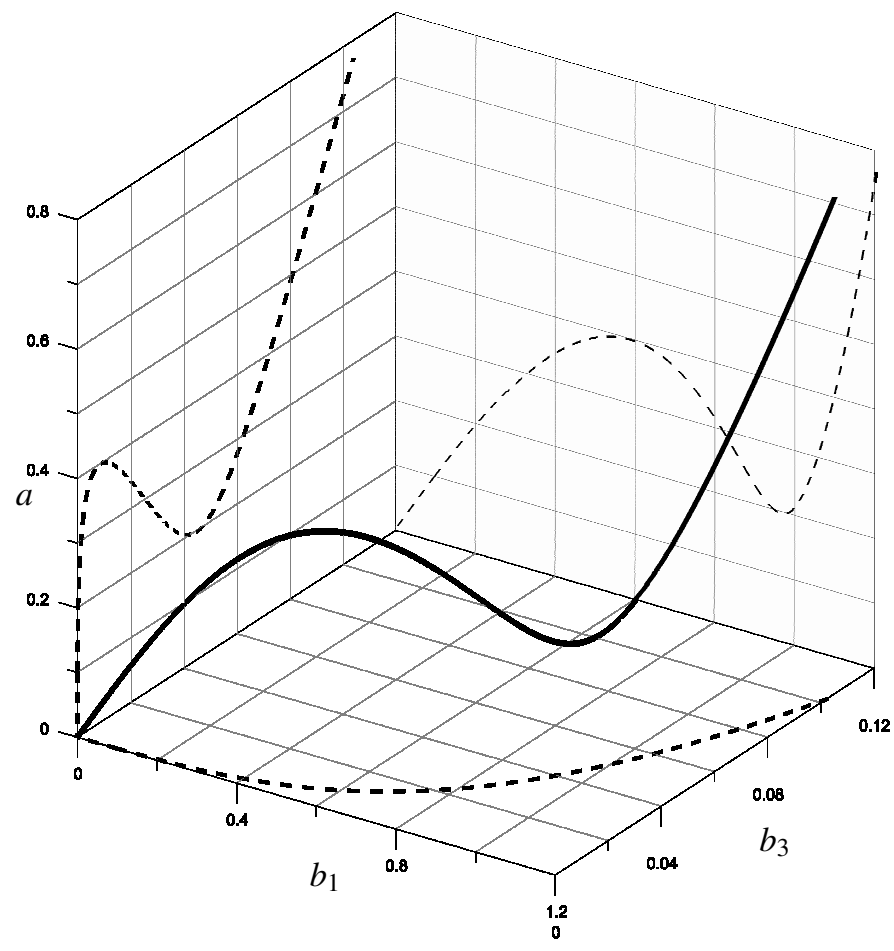

Figure 7. Typical nonlinear manifold for a NES-controlled system.

down at these singular points. To overcome this limitation, a further step must be accomplished in the perturbation analysis.

After the equation for $\mathbf{x}_{1}$ is solved and substituted in the higher-order equation for the NES (not shown for brevity), a solution of the form:

$$
z_{1}=\sum_{k=1}^{\infty} B_{1 k}\left(t_{1}, t_{2}, \ldots\right) \mathrm{e}^{i k \omega t_{0}}+c . c .
$$

is sought for via the HBM, which leads to differential equations of the type:

$$
\mathrm{O}(m, \xi) d_{1} \mathbf{B}_{1}=\mathbf{g}_{1}\left(A, \mathbf{B}_{1} ; \mu\right)
$$

where $\mathbf{B}_{1}:=\left(B_{11}, B_{12}, \ldots\right)$ and $\mathrm{O}(m, \xi)$ denotes coefficients of the order of the NES mass and damping coefficients, all close to zero. Here, therefore, the singular nature of the system clearly reveals itself.

By coming back to the original time, and by letting $\mathbf{B}:=$ $\mathbf{B}_{0}+\mathbf{B}_{1}$, we finally have:

$$
\begin{aligned}
& \dot{A}=f(A, \mathbf{B} ; \mu) \\
& \varepsilon \dot{\mathbf{B}}=\mathbf{g}(A, \mathbf{B} ; \mu)
\end{aligned}
$$

If just a harmonic were used in the HBM, then the system would be four-dimensional, according to the CM theory; however, due to the singularity of the system, which call for introducing several amplitudes, the dimension is higher.

It should be remarked that, differently from the Complexification procedure by Manevitch [32], usually adopted in literature to tackle this problem, the HBM has been used here just to solve one equation, namely the NES-equation, and not for the whole system.

The singular bifurcation equations (38) govern the wellknown relaxation oscillations, or 'Strongly Modulated Responses (SMR)' [33]. They consist in alternate slow and fast regimes. Indeed, when the system moves close to the (parameter dependent) manifold of equation $\mathbf{g}(A, \mathbf{B} ; \mu)=\mathbf{0}$, i.e. when $\mathbf{g}(A, \mathbf{B} ; \mu)=$ $\mathrm{O}(\varepsilon)$, then $\dot{\mathbf{B}}=\mathrm{O}(1)$, i.e. the motion is slow; when, in contrast, the system is far from the manifold, i.e. $\mathbf{g}(A, \mathbf{B} ; \mu)=\mathrm{O}(1)$, then $\dot{\mathbf{B}}=\mathrm{O}\left(\varepsilon^{-1}\right)$, i.e. the motion is fast.

The equations (38) were used in [11] to investigate the effectiveness of the NES in controlling the oscillations consequent to a Hopf bifurcation of a two d.o.f.'s airfoil, capable to vertically translate and to rotate. A single d.o.f. NES was attached to it, either windward or leeward. The analysis of the bifurcation equations led to the results displayed in Fig 8, where continuous lines denotes periodic solution for the original system (with and without NES) and dots numerical solutions. A small region of quasi-period solutions, consequent to secondary Hopf bifurcations, was also found (the curves denote the $\mathrm{min} / \mathrm{max}$ amplitudes). It is seen that the NES has always a beneficial linear effect, since it shifts forward the critical wind velocity $\mu$. However, while the first arrangement produces subcritical attractors, the second one entails a supercritical behavior, always reducing the amplitude of the limit-cycle.

Two phase-portraits in the amplitude-plane are reported in Fig 9, corresponding to the vertical lines in Fig 8. They show that (Fig 9-a) the stable periodic solution on the manifold is attractive of the system dynamics; in contrast (Fig 9-b), the unstable periodic motion existing between the two Hopf secondary bifurcations produces a limit cycle on the $(a, b)$-plane, which occurs around the manifold.

\subsection{Externally excited systems}

Nonlinear Energy Sinks are also employed to passively control systems under external excitation [33]; the MSM-HB also works for them [12]. As an example, let us consider a Hamiltonian system, slightly damped, subject to a harmonic excitation of frequency $\Omega$ and amplitude $\delta$, having equation:

$$
\begin{aligned}
& \dot{\mathbf{x}}=\mathbf{J}(\mu) \mathbf{x}+\mathbf{n}_{3}(\mathbf{x}, \mathbf{x}, \mathbf{x})+\ldots-\left(\xi \dot{z}+\kappa z^{3}\right) \hat{\mathbf{r}}+\delta \mathbf{f} \cos \Omega t \\
& m\left(\ddot{z}-\mathbf{r}^{T} \ddot{\mathbf{x}}\right)+\xi \dot{z}+\kappa z^{3}=0
\end{aligned}
$$

Here $\mathbf{J}(\mu)=\mathbf{J}_{0}+\mu \mathbf{J}_{1}$, where $\mathbf{J}_{0}$ is the Jacobian matrix of an Hamiltonian system, admitting all non-zero eigenvalues $\pm \omega_{j}$, and $\mu:=(\sigma, \xi)$ is a vector of small parameters, including stiffness and damping coefficients. Moreover, the external frequency $\Omega$ is assumed to be resonant with a natural frequency $\omega_{1}$, and other forms of resonance are excluded.

By rescaling the external force as $\delta \rightarrow \varepsilon \delta$ and using the same arguments for the autonomous system, we obtain perturbation equations like (30) and (31), this latter accounting for the new term:

$$
\begin{gathered}
\left(d_{0}-\mathbf{J}_{0}\right) \mathbf{x}_{1}=-d_{1} \mathbf{x}_{0}+\mu \mathbf{J}_{1} \mathbf{x}_{0}+\mathbf{n}_{3}\left(\mathbf{x}_{0}, \mathbf{x}_{0}, \mathbf{x}_{0}\right) \\
-\left(\xi d_{0} z_{0}+\kappa z_{0}^{3}\right) \hat{\mathbf{r}}+\frac{1}{2} \delta \mathbf{f}\left(\mathrm{e}^{i \Omega t_{0}}+\mathrm{e}^{-i \Omega t_{0}}\right) \\
m d_{0}^{2} z_{0}+\xi d_{0} z_{0}+\kappa z_{0}^{3}=m \mathbf{r}^{T} \ddot{d}_{0}^{2} \mathbf{x}_{0}
\end{gathered}
$$




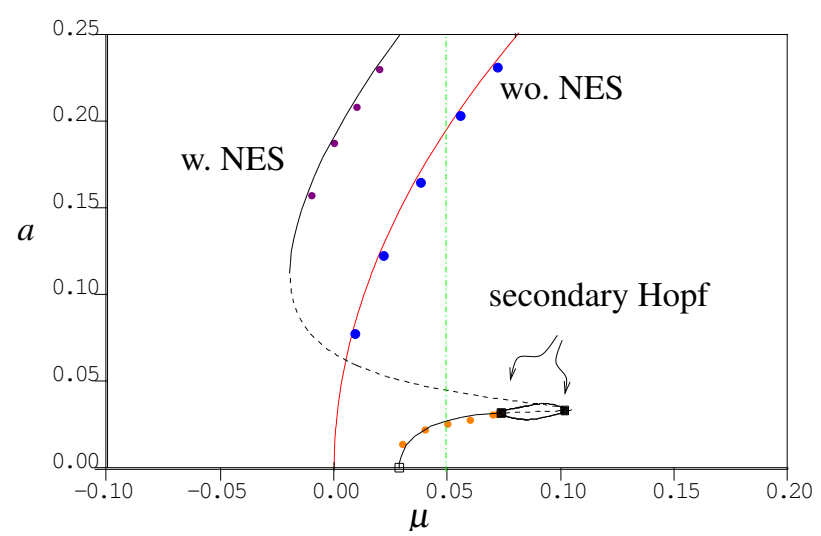

(a)

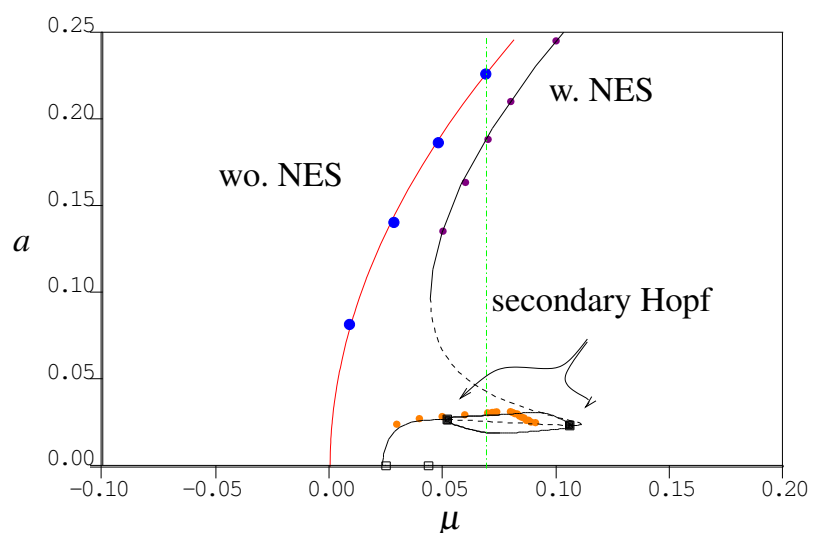

(b)

Figure 8. Periodic and quasi-periodic motions for an airfoil equipped with a NES [11], put (a) windward, and (b) leeward.

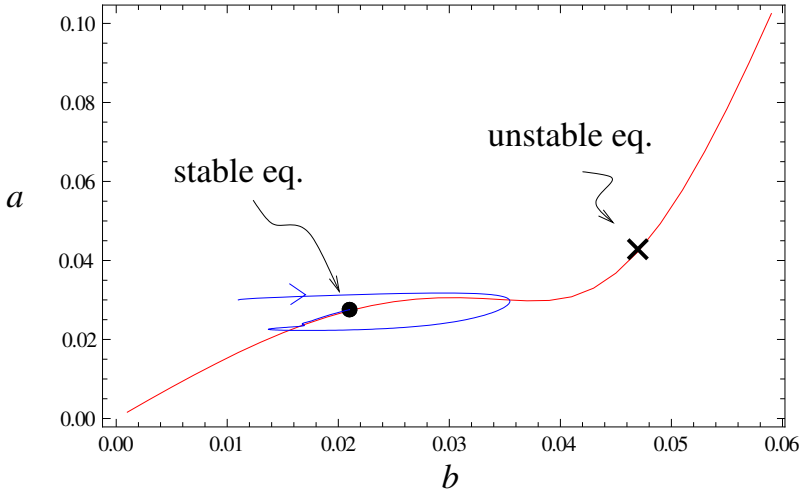

(a)

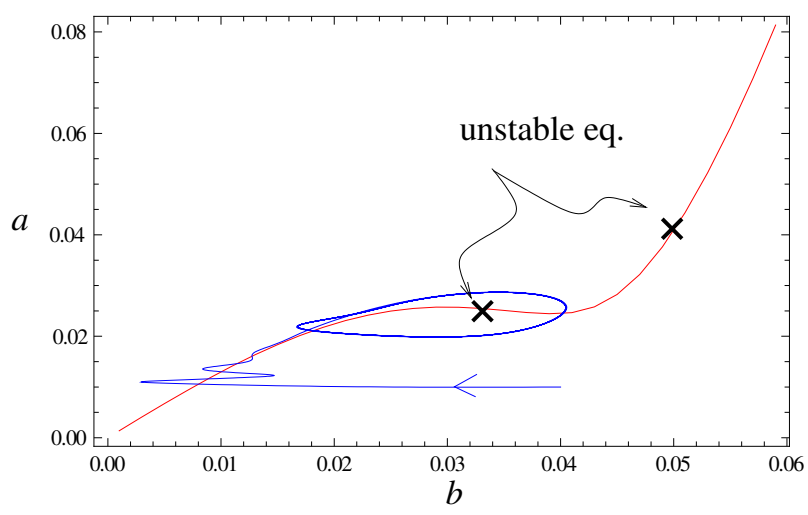

(b)

Figure 9. Phase-portrait for the airfoil [11], at the cross-sections marked in Fig 8: (a) $\mu=0.05$, (b) $\mu=0.07$.

Therefore, by using the generating solution (32), which takes into account only the natural mode involved in the resonance, and repeating the same steps as before, the following bifurcation equations are obtained, identical to Eqs (38), but including $\delta$ among the parameters:

$$
\begin{aligned}
& \dot{A}=f(A, \mathbf{B} ; \mu, \boldsymbol{\delta}) \\
& \varepsilon \dot{\mathbf{B}}=\mathbf{g}(A, \mathbf{B} ; \boldsymbol{\mu}, \boldsymbol{\delta})
\end{aligned}
$$

These equations have been used in [12] to analyze the response of a single d.o.f. system with a NES attached; some results are reported in Fig. 10. Fig. 10-a shows the amplitude-frequency curve; an unstable periodic motion (yellow circle) exists between secondary Hopf points (black squares). When the motion is plotted on the amplitude plane (Fig. 10-b) SMR are observed, occurring around the unstable periodic motion. The time-histories of the amplitude (Fig. 10-c) and that of the displacement $x$ (Fig. 10-d) show the relaxation oscillations.

\section{Conclusions}

We discussed how to adapt the Multiple Scale Method in order to solve 'difficult' bifurcation problems. We obtained the following main results.
1. When the system is defective at the bifurcation, i.e. the center eigenvalue does not possess a complete set of eigenvectors, fractional power expansions and fractional times of order $\varepsilon^{1 / m}$ must be used, with $m$ the algebraic multiplicity of the eigenvalue. By systematically enforcing solvability conditions at each order, and then recombining them on the true time-scale, an $m$-order differential equation for the amplitude is drawn.

2. When the system is partially defective at the bifurcation, i.e. defective and non-defective eigenvalues interact, then fractional powers must be used, although some of the derivatives of the amplitudes can be zero, this denoting that the dynamics is of slow-fast type. Consequently, the bifurcation equations are differential equations of different order.

3. When the system is, in addition, singular, as it happens for NES-equipped systems, integer powers must be used, in conjunction with the Harmonic Balance Method to solve singular equations. The bifurcation equations are found to be in the form of singular equations, exhibiting relaxation oscillations.

Several applications have been shown, to display mechanical phenomenon and to test the effectiveness of the proposed meth- 


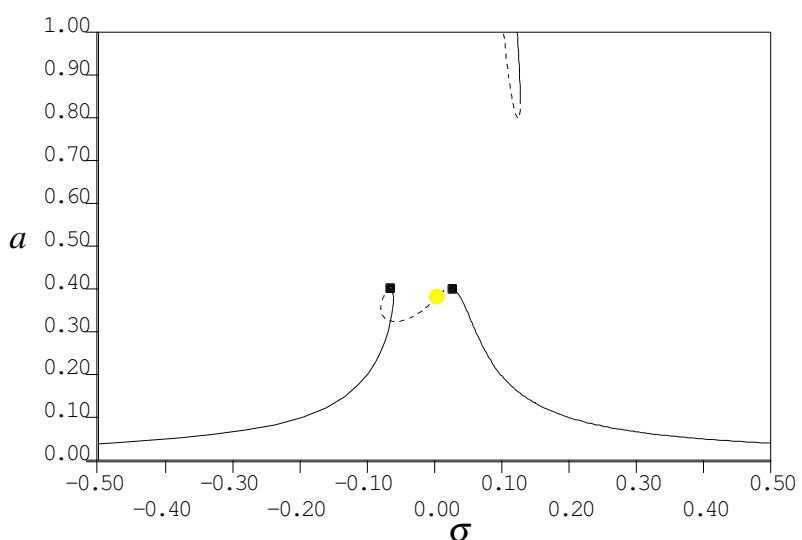

(a)

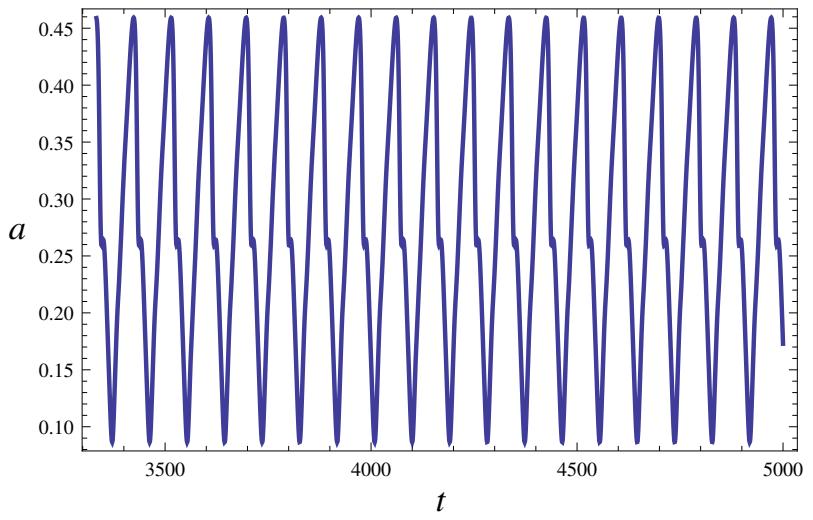

(c)

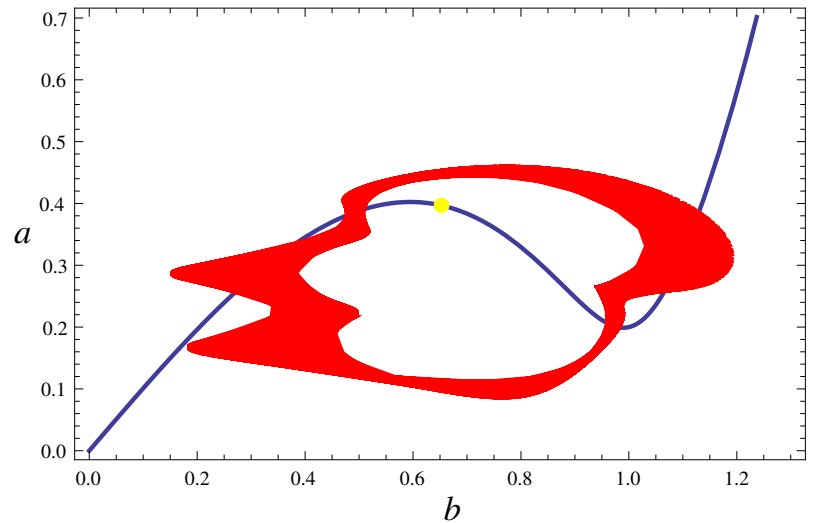

(b)

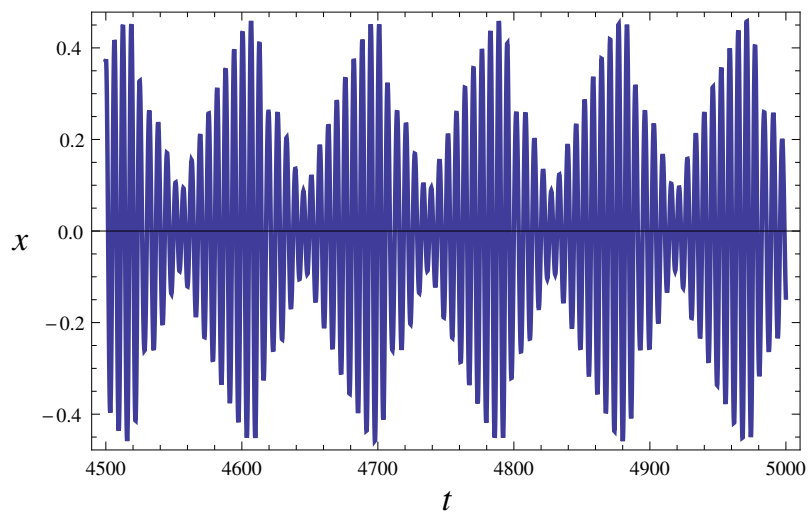

(d)

Figure 10. Response of a single-d.o.f. system equipped with NES to external excitation [12]: (a) amplitude-frequency response; (b) phase-portrait; (c), (d) time-histories.

ods. They include discrete and continuous systems, autonomous, parametrically excited and externally excited systems. Moreover, the algorithm was able to explain the transition between two bifurcations of different codimensions, when a parameter is varied.

\section{Acknowledgments}

This work is supported by the Italian Ministry of University: PRIN 2010-2011 program 2010MBJK5B.

\section{References}

[1] J. Guckenheimer and P. Holmes. Nonlinear Oscillations, Dynamical Systems, and Bifurcation of Vector Fields. Springer-Verlag, New York, 1983.

[2] A.H. Nayfeh and D.T. Mook. Nonlinear oscillations. John Wiley, New York, 1979.

[3] A. Luongo, A. Di Egidio, and A. Paolone. Computational Problems in Multiple Scale Analysis, pages 1-31. Recent Research Developments in Sound and Vibration Transworld Research Network, Kerala - India, 2002.
[4] A. Luongo, A. Di Egidio, and A. Paolone. Multiple Scale Bifurcation Analysis for Finite-Dimensional Autonomous Systems, pages 161-201. Recent Research Developments in Sound and Vibration - Transworld Research Network, Kerala - India, 2002.

[5] A. Luongo and A. Paolone. On the reconstitution problem in the multiple time scale method. Nonlinear Dynamics, 19(2):133-156, 1999.

[6] A. Luongo. A unified perturbation approach to static/dynamic coupled instabilities of nonlinear structures. Thin-Walled Structures, 48(10-11):744-751, 2010.

[7] A. Luongo, A. Di Egidio, and A. Paolone. Multiple time scale analysis for bifurcation from a multiple-zero eigenvalue. AIAA Journal, 41(6):1143-1150, 2003.

[8] A. Luongo, A. Di Egidio, and A. Paolone. Multiscale analysis of defective multiple-Hopf bifurcations. Computers and Structures, 81(31-32):2705-2722, 2004.

[9] A. Luongo and D. Zulli. A paradigmatic system to study the transition from zero/Hopf to double-zero/Hopf bifurcation. Nonlinear Dynamics, 70(1):111-124, 2012. 
[10] A. Luongo, I. Arkhipova, and A.P. Seyranian. Vibrational stabilization of upper statically unstable position of double pendulum. Journal of Sound and Vibration, 331:457-469, 2012.

[11] A. Luongo and D. Zulli. Aereoelastic instability analysis of NES-controlled systems via a mixed Multiple Scale/Harmonic Balance algorithm. Journal of Vibration and Control, In press, 2013.

[12] A. Luongo and D. Zulli. Dynamic analysis of externally excited NES-controlled systems via a mixed Multiple Scale/Harmonic Balance algorithm. Nonlinear Dynamics, 70(3):2049-2061, 2012.

[13] A. Luongo and A. Paolone. Perturbation methods for bifurcation analysis from multiple nonresonant complex eigenvalues. Nonlinear Dynamics, 14(3):193-210, 1997.

[14] A. Luongo and A. Paolone. Multiple scale analysis for divergence-hopf bifurcation of imperfect symmetric systems. Journal of Sound and Vibration, 218(3):527-539, 1998.

[15] A. Luongo, A. Paolone, and A. Di Egidio. Multiple time scales analysis for 1:2 and 1:3 resonant Hopf bifurcations. Nonlinear Dynamics, 34(3-4):269-291, 2003.

[16] A. Paolone, M. Vasta, and A. Luongo. Flexural-torsional bifurcations of a cantilever beam under potential and circulatory forces: Part I nonlinear model and stability analysis. International Journal of Non-Linear Mechanics, 41(4):586594, 2006.

[17] A. Paolone, M. Vasta, and A. Luongo. Flexural-torsional bifurcations of a cantilever beam under potential and circulatory forces: Part II post-critical analysis. International Journal of Non-Linear Mechanics, 41(4):595-604, 2006.

[18] Di Egidio, A. Luongo, and A. Paolone. Linear and nonlinear interactions between static and dynamic bifurcations of damped planar beams. International Journal of Non-Linear Mechanics, 42(1):88-98, 2007.

[19] A. Luongo and A. Di Egidio. Bifurcation equations through multiple-scales analysis for a continuous model of a planar beam. Nonlinear Dynamics, 41(1-3):171-190, 2005.

[20] A. Luongo and A. Di Egidio. Divergence, Hopf and doublezero bifurcations of a nonlinear planar beam. Computers and Structures, 84(24-25):1596-1605, 2006.

[21] A. Luongo and F. D'Annibale. Bifurcation analysis of damped visco-elastic planar beams under simultaneous gravitational and follower forces. International Journal of Modern Physics B, 26(25), 2012.

[22] A. Luongo and F. D'Annibale. Double zero bifurcation of nonlinear viscoelastic beams under conservative and nonconservative loads. International Journal of Non-Linear Mechanics, In press, 2013.
[23] M.V. Keldysh. On eigenvalues and eigenfunctions of certain classes of not self-adjoint equations. Doklady Akademii Nauk SSRR, 77 In Russian(1):111-124, 1955.

[24] O. Kirillov and A Seyranian. Collapse of the Keldysh chains and stability of continuous non-conservative systems. SIAM Journal on Applied Mathematics, 64(4):1383-1407, 2004.

[25] J.J. Thomsen. Theories and experiments on the stiffening effect of high-frequency excitation for continuous elastic systems. Journal of Sound and Vibrations, 260(4):117-139, 2003.

[26] A.A. Seyranian and A.P. Seyranian. The stability of an inverted pendulum with a vibrating suspension point. Journal of Applied Mathematics and Mechanics, 70:754-761, 2006.

[27] A.F. Vakakis, O.V. Gendelman, L.A. Bergman, D.M. McFarland, G. Kerschen, and Y.S. Lee. Nonlinear Targeted Energy Transfer in Mechanical and Structural Systems I. Springer-Verlag, New York, 2008.

[28] A.F. Vakakis, O.V. Gendelman, L.A. Bergman, D.M. McFarland, G. Kerschen, and Y.S. Lee. Nonlinear Targeted Energy Transfer in Mechanical and Structural Systems II. Springer-Verlag, New York, 2008.

[29] V. Gattulli, F. Di Fabio, and A. Luongo. One to one resonant double hopf bifurcation in aeroelastic oscillators with tuned mass dampers. Journal of Sound and Vibration, 262(2):201$217,2003$.

[30] V. Gattulli, F. Di Fabio, and A. Luongo. Nonlinear tuned mass damper for self-excited oscillations. Wind and Structures, 7(4):251-264, 2004.

[31] X. Jiang, D.M. McFarland, L.A. Bergman, and A.F. Vakakis. Steady State Passive Nonlinear Energy Pumping in Coupled Oscillators: Theoretical and Experimental Results. Nonlinear Dynamics, 33:87-102, 2003.

[32] L. Manevitch. The description of localized normal modes in a chain of nonlinear coupled oscillators using complex variables. Nonlinear Dynamics, 25:95-109, 2001.

[33] Y. Starosvetsky and O.V. Gendelman. Response regimes of linear oscillator coupled to nonlinear energy sink with harmonic forcing and frequency detuning. Journal of Sound and Vibration, 315:746-765, 2008. 